\title{
Inversion of GPS meteorology data
}

\author{
K. Hocke \\ Institut für Meteorologie und Geophysik, Universität Graz, A-8010 Graz, Halbärthgasse 1, Austria \\ Received: 3 July 1996 / Revised: 6 November 1996 / Accepted: 28 November 1996
}

\begin{abstract}
The GPS meteorology (GPS/MET) experiment, led by the Universities Corporation for Atmospheric Research (UCAR), consists of a GPS receiver aboard a low earth orbit (LEO) satellite which was launched on 3 April 1995. During a radio occultation the LEO satellite rises or sets relative to one of the 24 GPS satellites at the Earth's horizon. Thereby the atmospheric layers are successively sounded by radio waves which propagate from the GPS satellite to the LEO satellite. From the observed phase path increases, which are due to refraction of the radio waves by the ionosphere and the neutral atmosphere, the atmospheric parameter refractivity, density, pressure and temperature are calculated with high accuracy and resolution $(0.5-1.5 \mathrm{~km})$. In the present study, practical aspects of the GPS/MET data analysis are discussed. The retrieval is based on the Abelian integral inversion of the atmospheric bending angle profile into the refractivity index profile. The problem of the upper boundary condition of the Abelian integral is described by examples. The statistical optimization approach which is applied to the data above $40 \mathrm{~km}$ and the use of topside bending angle profiles from model atmospheres stabilize the inversion. The retrieved temperature profiles are compared with corresponding profiles which have already been calculated by scientists of UCAR and Jet Propulsion Laboratory (JPL), using Abelian integral inversion too. The comparison shows that in some cases large differences occur ( $5 \mathrm{~K}$ and more). This is probably due to different treatment of the upper boundary condition, data runaways and noise. Several temperature profiles with wavelike structures at tropospheric and stratospheric heights are shown. While the periodic structures at upper stratospheric heights could be caused by residual errors of the ionospheric correction method, the periodic temperature fluctuations at heights below $30 \mathrm{~km}$ are most likely caused by atmospheric waves
\end{abstract}

Present address: Communications Research Laboratory, Upper Atmosphere Section, 4-2-1 Nukui- Kita, Koganei- shi, Tokyo 184, Japan, hocke@ crl.go.jp (vertically propagating large-scale gravity waves and equatorial waves).

\section{Introduction}

The radio occultation technique was applied for investigation of planetary atmospheres and ionospheres since the sixties (e.g., Kliore et al., 1965; Fjeldbo and Eshelman, 1965). The radio waves transmitted by spacecraft (e.g., Mariner, Voyager) were received at the Earth. When the radio path transected the planetary atmosphere and ionosphere during an occultation, height profiles of atmospheric and ionospheric parameters could be retrieved from the observed phase path increases and amplitude variations.

The potential of the occultation technique for remote sensing of the Earth's atmosphere was experimentally and theoretically investigated, mainly by scientists from Russia (e.g., Gurvich et al., 1982; Sokolovskiy, 1990; Gorbunov, 1988). The installation of the global positioning system (GPS) in the last decade now allows the exact determination of satellite positions and velocities which is required for the accurate measurement of the small phase path increase caused by the neutral atmosphere. Theoretical considerations and simulations promised that the radio occultation method can provide temperature profiles with an accuracy of $1 \mathrm{~K}$ and less in the troposphere and stratosphere (Gorbunov and Sokolovskiy, 1993).

The GPS/MET experiment is the first phase of the plan to develop an observation system of low earth orbit satellites which will provide continuous data on the vertical distribution of density, pressure, temperature and probably humidity in the atmosphere all over the globe. The experiment was started on 3 April 1995 with the launch of the small research satellite MicroLab1 in a low earth orbit. The database of the collected radio occultation data and retrieved atmospheric profiles can 


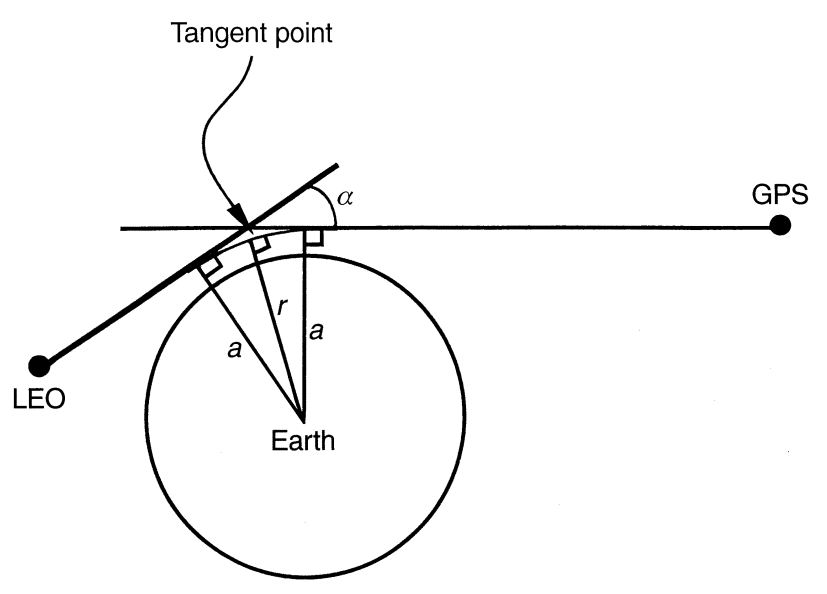

Fig. 1. Occultation geometry defining the impact parameter, $a$, the bending angle, $\alpha$ and the radius to the ray tangent point, $r$. (from Kursinski et al., 1996a)

be accessed via internet (http://pocc.gpsmet.ucar.edu). Per day 100-200 occultation events which are arbitrary distributed over the globe are available. In Fig. 1 the geometry of the experiment is sketched. Detailed descriptions of the experiment and the data analysis were given by Ware et al. (1996), Hajj et al. (1995) and Kursinski et al. (1996a).

\section{Data analysis}

\subsection{Derivation of the atmospheric bending angle}

The retrieval is based on the Abelian integral inversion, like those retrievals made by scientists of UCAR and JPL (Ware et al., 1996; Kursinski et al., 1996a). Before the inversion, the atmospheric bending angle profile is calculated from the measured time series of phase path increases $\Phi_{1}, \Phi_{2}$ and of the satellites velocities and positions. $\Phi_{1}$ and $\Phi_{2}$ are the phase path increases (total ray path length less the vacuum path length between the transmitter and receiver) of the two GPS carrier frequencies, $f_{1}$ and $f_{2}$. There are two linear ionospheric correction methods for the removal of the ionospheric contribution:

1. The traditional linear correction of phases

$$
\Phi_{L C}(t)=\frac{f_{1}^{2} \Phi_{1}(t)-f_{2}^{2} \Phi_{2}(t)}{f_{1}^{2}-f_{2}^{2}}
$$

$\Phi_{L C}$ : linear corrected phase path increase without ionospheric contribution

$$
t \text { : time, }
$$

2. The linear correction of bending angles (Vorob'ev and Krasil'nikova, 1993).

The linear correction method of the bending angles provides, in particular for ionospheres with high electron densities (daytime, solar maximum), obviously better results than the traditional correction of phases (Vorob'ev and Krasil'nikova, 1993; Melbourne et al., 1994).
In the present work the linear correction of bending angles is applied. First of all the Doppler shifts of the two GPS carrier frequencies are calculated:

$\frac{\Delta f_{i}}{f_{i}}=\frac{1}{c} \frac{d \Phi_{i}}{d t}, \quad i=1,2$,

where $c$ is the vacuum speed of light. In Fig. 2 the height profile of the $f_{1}$ Doppler shift (long dashed curve with label, total) is shown for one occultation event. Since the phase path increases were subtracted by the vacuum path lengths, the vacuum Doppler shift is not included in the depicted Doppler shift. The contribution of the neutral atmosphere to the Doppler shift was estimated by differentiation of the linear corrected phase path increase $\Phi_{L C}$ (Eq. 1). The ionospheric contribution is then the difference between the total Doppler shift and the neutral Doppler shift. While at heights above around $45 \mathrm{~km}$ the refraction by the ionosphere becomes dominant, at heights below $40 \mathrm{~km}$ the refraction by the neutral atmosphere dominates and increases exponentially with decreasing height.

Knowing the positions and the velocities of the GPS and LEO satellites during the occultation event, the Doppler shift equation (Vorob'ev and Krasil'nikova, 1994, formula 5) is solved for the $f_{1}$ and $f_{2}$ Doppler shift separately in order to calculate the bending angles $\alpha_{1}$ and $\alpha_{2}$ as function of the impact parameters $a_{1}$ and $a_{2}$ (Fig. 1).

The atmospheric bending angle $\alpha$ is then obtained by the linear correction method of Vorob'ev and Krasil'nikova (1993):

$\alpha(a)=\frac{f_{1}^{2} \alpha_{1}(a)-f_{2}^{2} \alpha_{2}(a)}{f_{1}^{2}-f_{2}^{2}}$.

It is important that $\alpha_{1}$ and $\alpha_{2}$ are matched to the same impact parameter $a$. In practise, the $\alpha_{2}(t)$ and $a_{2}(t)$ series

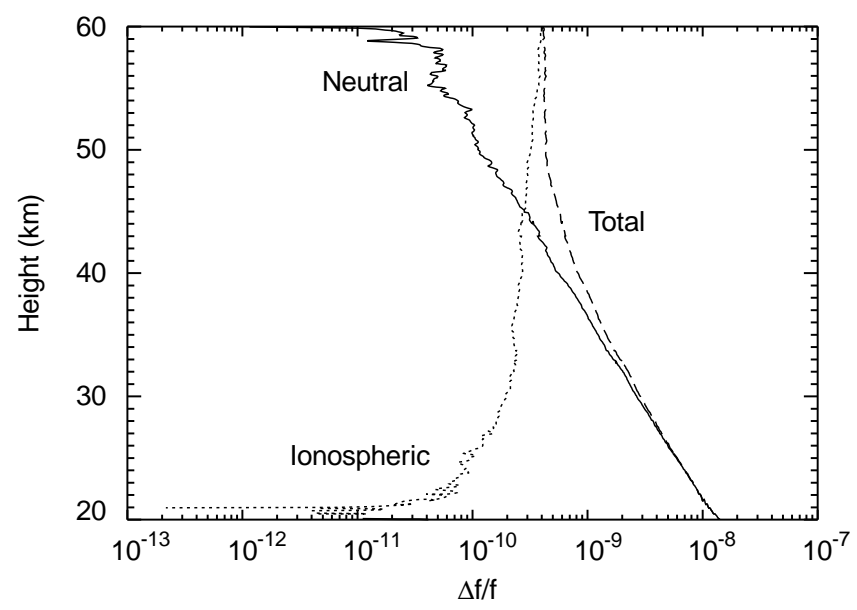

Fig. 2. Doppler shift (without vacuum Doppler shift) as function of height for one occultation event (0031-05.17-95.298). The solid line is the Doppler shift caused by the refraction of the neutral atmosphere, the dotted line is the Doppler shift caused by the ionosphere and the dashed line is the total measured Doppler shift. Below $30 \mathrm{~km}$ height the ionospheric contribution to the Doppler shift is 1-2 magnitudes less than the neutral contribution 


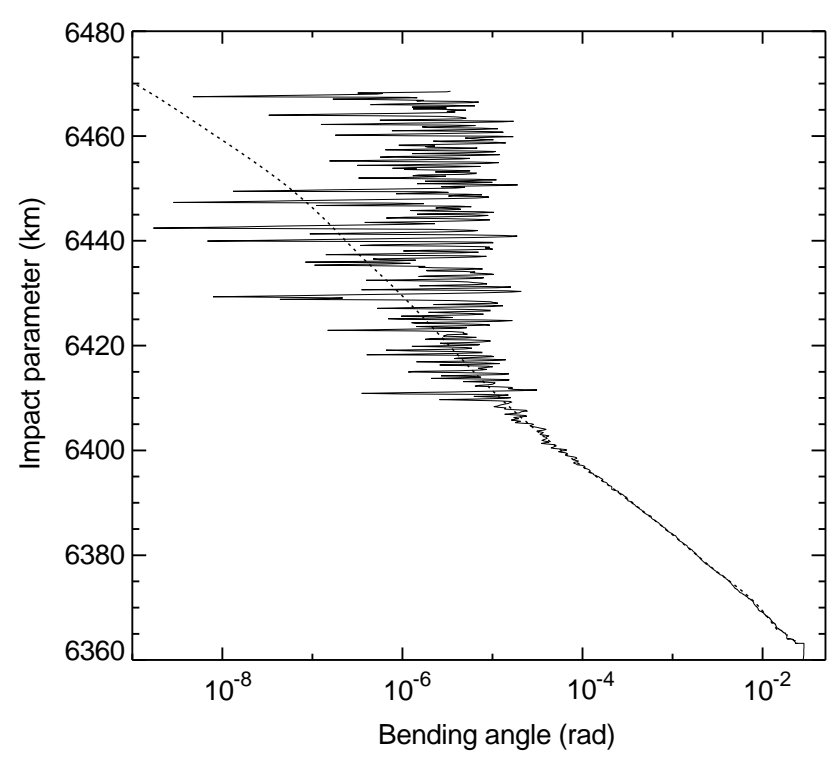

Fig. 3. Bending angle of the neutral atmosphere as function of height for one occultation event. The solid line is the bending angle retrieved from the GPS/MET measurements (event 0030-04.27-95.182). The dotted line is calculated from the density profile of the MSISE-90 model at the place and time of the occultation

have to be interpolated. Thereafter the $\alpha_{2}\left(a_{1}\right)$ values, belonging to $\alpha_{1}\left(a_{1}\right)$, are selected. By this construction the impact parameter $a$ is equal to $a_{1}$. In Fig. 3 the atmospheric bending angle is depicted as function of the impact parameter for a typical radio occultation event. The solid line denotes the bending angle $\alpha$ derived from the GPS/MET measurement, while the dotted line is due to the bending angle $\alpha_{m}$ calculated from the density profile of the MSISE-90 atmosphere model (Hedin, 1991) at the occultation place and time. At heights beyond $50 \mathrm{~km}$ (this corresponds to impact parameter $6410 \mathrm{~km}$ in the figure) the relative error of the measured bending angle is greater than $100 \%$. For the inversion of the bending angle profile it is better to use a combination of the model bending angle $\alpha_{m}$ at heights above $50-60 \mathrm{~km}$ and the measured bending angle $\alpha$ at heights below.

\subsection{Abelian integral inversion}

The bending angle can be derived from the refractivity index profile $\mu(a)$ by following integral (Fjeldbo et al., 1971):

$\alpha\left(a_{0}\right)=2 a_{0} \int_{a=a_{0}}^{a=\infty} \frac{d \ln \mu(a)}{d a} \frac{1}{\sqrt{a^{2}-a_{0}^{2}}} d a$,

whereby the impact parameter $a_{0}$ corresponds to the radius at the tangent point $r_{0}=a_{0} / \mu\left(a_{0}\right)$ for the ray of closest approach. This equation can be inverted by the Abelian transformation, so that for a given atmospheric bending angle profile $\alpha(a)$ the refractivity index profile $\mu(a)$ is calculated by $\ln \left(\mu\left(a_{0}\right)\right)=\frac{1}{\pi} \int_{a=a_{0}}^{a=\infty} \frac{\alpha(a)}{\sqrt{a^{2}-a_{0}^{2}}} d a$.

The Abelian integral inversion was described in detail in Appendix C of Fjeldbo et al. (1971). For the numerical solution, the integral of Eq. (5) can be integrated by parts in order to avoid the pole of $\left(a^{2}-a_{0}^{2}\right)^{-1 / 2}$ (Fjeldbo et al., 1971):

$\ln \left(\mu\left(a_{0}\right)\right)=\frac{1}{\pi} \int_{\alpha=\alpha\left(a_{0}\right)}^{\alpha=0} \ln \left\{\frac{a(\alpha)}{a_{0}}+\sqrt{\left(\frac{a(\alpha)}{a_{0}}\right)^{2}-1}\right\} d \alpha$.

By the same procedure Eq. (4) gives

$\alpha\left(a_{0}\right)=2 a_{0} \int_{a=a_{0}}^{a=\infty} \frac{d^{2} \ln (\mu(a))}{d a^{2}} \ln \left\{\frac{a}{a_{0}}+\sqrt{\left(\frac{a}{a_{0}}\right)^{2}-1}\right\} d a$.

In principle the atmospheric parameters of refractivity $N$, density $\rho$, pressure $p$ and temperature $T$ are determined by following equations (e.g., Ware et al., 1996), if water vapor is negligible. Water vapor can be neglected in cold regions of the troposphere and stratosphere with temperatures lower than $\sim 250 \mathrm{~K}$.

$r=a / \mu(a), \quad h=r-r_{E}$

$N(h)=10^{6}(\mu(h)-1)$

$\rho(h)=\frac{M}{c_{1} R} N(h)$

$p(h)=g(h) \int_{h}^{\infty} \rho\left(h^{\prime}\right) d h^{\prime}$

$T(h)=\frac{M}{R} \frac{p(h)}{\rho(h)}$

$r$ : radius at the tangent point (Fig. 1)

$r_{E}$ : Earth's radius at the occultation place

$c_{1}$ : constant factor $=77.6 \mathrm{~K} / \mathrm{mbar}$

$M$ : mean molecular mass of air $(28.964 \mathrm{~kg} / \mathrm{kmol}$ at

heights below $80 \mathrm{~km}$ )

$R$ : universal gas constant $=8314 \mathrm{~J} /(\mathrm{K} \mathrm{kmol})$

$g(h)$ : gravity $=g_{0}\left(r_{E} /\left(r_{E}+h\right)\right)^{2}$

$g_{0}$ : gravity at the ground $=9.807 \mathrm{~m} / \mathrm{s}^{2}$

\subsection{Upper boundary condition}

As shown in Fig. 3 there are difficulties resulting from the fact that at higher altitudes above $50-60 \mathrm{~km}$ the error of the bending angle is greater than $100 \%$. The error is caused by residual ionospheric errors becoming more and more important with increasing height since the atmospheric bending angle exponentially decreases. Furthermore there are limitations of the measurement: the path increase due to atmospheric refraction is at heights above $50 \mathrm{~km}$ less than $10 \mathrm{~cm}$. The limitations are due to thermal noise of the GPS receiver, clock errors and orbit uncertainty. 
With the help of Eqs. (7), (9) and (10), the bending angle profile $\alpha_{m}$ is calculated from the density profile of a model atmosphere at the occultation place and time (see Fig. 3, dotted line). The model atmosphere is represented by the MSISE-90 model of Hedin (1991). If $a_{I}$ denotes the upper boundary for the measured bending angle, Eq. (5) is now approximated as

$$
\begin{aligned}
\ln \left(\mu\left(a_{0}\right)\right) \approx \frac{1}{\pi} \int_{a_{0}}^{a_{I}} \frac{\alpha(a)}{\sqrt{a^{2}-a_{0}^{2}}} d a \\
\quad+\frac{1}{\pi} \int_{a_{I}}^{r_{E}+110 \mathrm{~km}} \frac{\alpha_{m}(a)}{\sqrt{a^{2}-a_{0}^{2}}} d a
\end{aligned}
$$

for $r_{E}<a_{0} \leq a_{I} . a_{I}$ is the initial impact parameter (corresponding to the initial height $h_{I}$ ) for the initialization of the integration of the measured bending angle $\left(r_{E}+45 \mathrm{~km} \leq a_{I} \leq r_{E}+60 \mathrm{~km}\right)$. The integration of the model bending angle stops at $110 \mathrm{~km}$ height which is more than 5 scale heights above the boundary $a_{I}$. For the temperature retrieval it is common to assume a temperature as boundary condition: $T_{I}=T_{M S I S E}\left(h_{I}\right)$ (e.g., Melbourne et al., 1994; Hajj et al., 1995). For $h \leq h_{I}$ Eqs. (9-12) are:

$$
\begin{aligned}
& N(h)=10^{6}(\mu(h)-1) \\
& \rho(h)=\frac{M}{c_{1} R} N(h) \\
& T(h)=\frac{M}{R} g(h) \frac{\int_{h}^{h_{I}} N\left(h^{\prime}\right) d h^{\prime}}{N(h)}+T_{I} \frac{N\left(h_{I}\right)}{N(h)} \\
& p(h)=p_{I}+g(h) \int_{h}^{h_{I}} \rho\left(h^{\prime}\right) d h^{\prime} \\
& \text { with } p_{I}=\frac{R}{M} \rho\left(h_{I}\right) T_{I} .
\end{aligned}
$$

The influence of a various choice of the initial height $h_{I}$ on the temperature profile is depicted in Fig. 4 (dotted lines) for one example. Especially at upper stratospheric heights different temperatures are retrieved for the different initial heights, which is not desired. The differences can be explained by the changing ionospheric residual error of the bending angle in that region. In some cases the choice of an initial height in a range of high bending angle error causes a failure of the inversion or significant temperature errors of the retrieved profile even in the lower stratosphere. By the statistical optimization approach the influence of the initial height selection on the temperature retrieval can be minimized, so that almost equal temperature profiles are retrieved for different initial heights.

\subsection{Statistical optimization approach}

The statistical optimization approach was introduced at the GPS/MET workshop in Tucson/Arizona by Sokolovskiy and Hunt (1996). In the following a brief description is given for the related approach which is used in the present study.

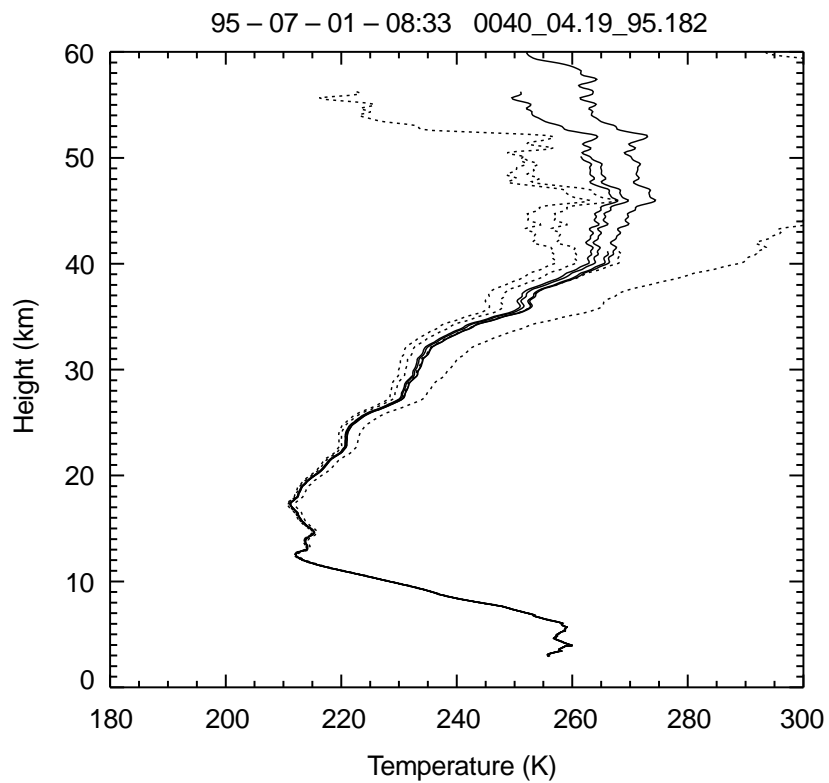

Fig. 4. Effect of different initialization heights of the Abelian integral inversion on the temperature profile. The dotted lines are due to the retrieval procedure without statistical optimization. The solid lines denote the curves derived with the statistical optimization approach for the bending angle data between $40 \mathrm{~km}$ and the initialization height

At first the noise of the measured bending angle profile $\alpha$ is estimated by the deviation from the model bending angle $\alpha_{m}$

$\sigma_{\text {noise }}=\alpha-\alpha_{m}$.

The expected magnitude of variations of the climatological bending angle is

$\sigma_{\text {signal }}=20 \% \alpha_{m}$.

Twenty percent is relatively arbitrary and was chosen by Sokolovskiy and Hunt (1996). In Fig. 5b $\sigma_{\text {noise }}$ and $\sigma_{\text {signal }}$ are shown for one example. While at heights below $40 \mathrm{~km}$ (corresponding to impact parameter around $6410 \mathrm{~km}$ ) the expected variations of the bending angle $\sigma_{\text {signal }}$ (solid line) are greater than $\sigma_{\text {noise }}$ (dotted line), $\sigma_{\text {noise }}$ is greater than $\sigma_{\text {signal }}$ at heights above $50 \mathrm{~km}$. It is obvious that there are bursts of noise at different heights (Fig. 5b). This can be due to disturbances of the radio ray by small-scale structures of the ionosphere which reduce the quality of the measurement (Kursinski et al., 1996b).

The weighting coefficient $C$ which is shown in Fig. 5a is given by

$C=\frac{1}{1+\left|\frac{\sigma_{\text {noise }}}{\sigma_{\text {signal }}}\right|}$.

The profile of the weighting coefficient may be used for the selection of the initial height. A weighting coefficient around $0.3-0.5$ seems to be appropriate. Of course the initial height can be also chosen at smaller weighting coefficient (e.g., 0.1, 0.2) but in this case the measured bending angle is strongly modified and almost equal to the model bending angle profile. The modification of the bending angle is 

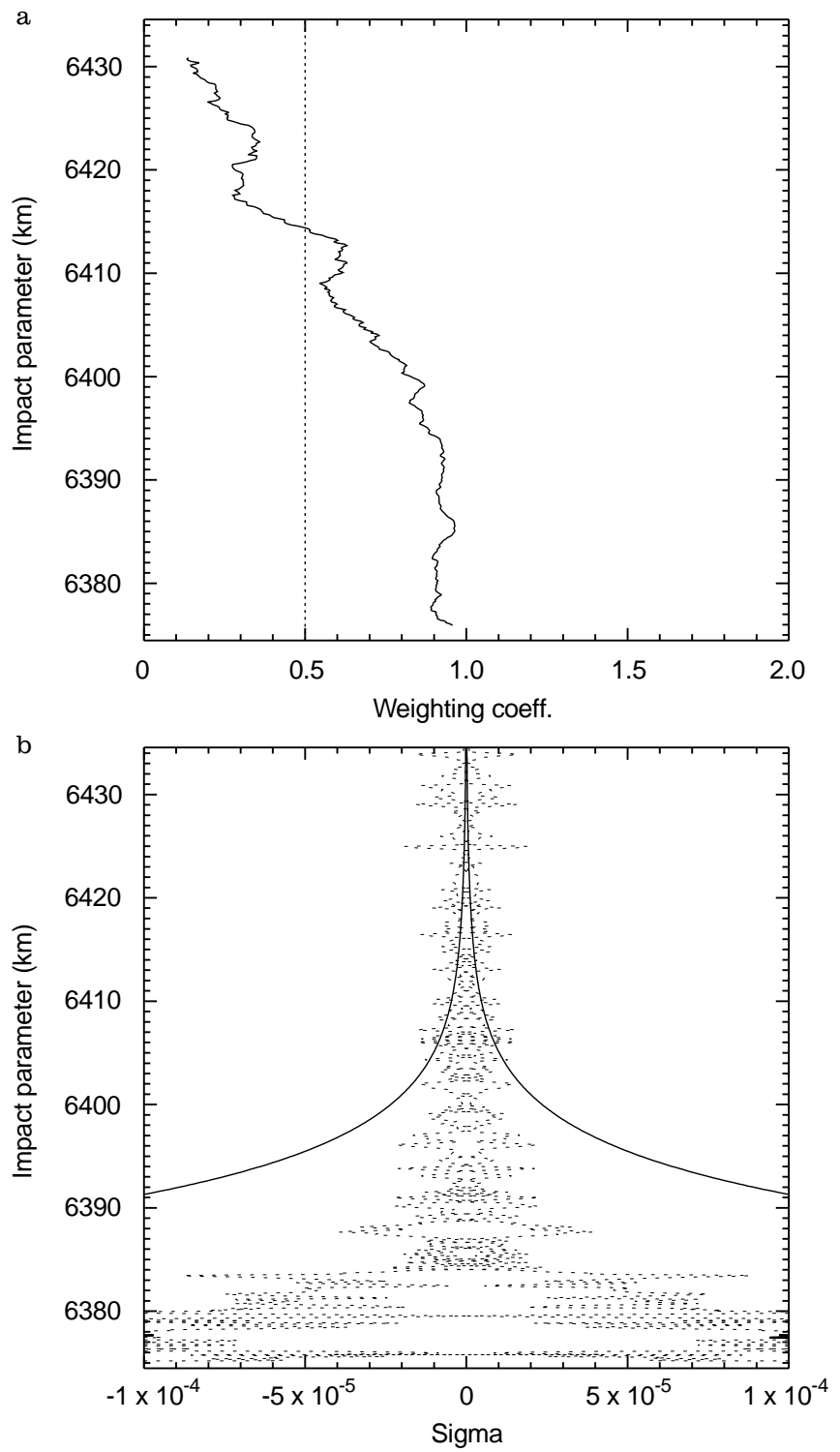

Fig. 5. a Weighting coefficient as function of impact parameter. b Comparison between the expected magnitude of variations of the climatological bending angle $\left(\sigma_{\text {signal }}\right.$, solid line $)$ and the noise of the measured bending angle $\left(\sigma_{\text {noise }}\right.$, dotted line $)$

$\alpha_{\text {new }}=\alpha_{m}+C \sigma_{\text {noise }}$.

The effect of this modification can be explained by regarding the limits: if $C \rightarrow 1$ (at lower altitudes) then $\alpha_{\text {new }} \rightarrow \alpha$, and if $C \rightarrow 0$ (at higher altitudes) then $\alpha_{\text {new }} \rightarrow \alpha_{m}$. Therefore, the transition from the measured bending angle profile $\alpha$ to the model bending angle $\alpha_{m}$ at heights beyond the upper boundary is smoothed. In this work the bending angle is only modified in the transition region between $40 \mathrm{~km}$ and the initial height $h_{I}$ (ca. 50-60 km). Figure 4 shows temperature profiles of the same occultation which were retrieved by using different initial heights. The dotted lines were calculated without statistical optimization and the solid lines with statistical optimization. It is obvious that the four solid lines show smaller differences than the dotted curves. The use of the bending angle data with high error at high altitudes produce errors in the retrieved temperature of around $2 \mathrm{~K}$ at $20 \mathrm{~km}$ height (difference of dotted lines at that height). The statistical optimization method avoids this error since the measured bending angle data above $40 \mathrm{~km}$ height is controlled by the model bending angle data.

\section{Discussion}

\subsection{Comparison of temperature profiles}

The described retrieval was supplemented by using the correction for the ellipsoidal shape of the Earth which was derived by Syndergaard and Hoeg (1996). This correction is applied in order to fulfill the spherical symmetry condition for the Abelian integral inversion. The retrieval was tested with simulation data of the $3 \mathrm{D}$ ray tracing model of Hoeg et al. (1996). If the ionosphere is absent in the simulation, the error of the retrieved temperature is around $0.2-0.5 \mathrm{~K}$ at heights below $40 \mathrm{~km}$. The small error might be caused by horizontal variations of the model atmosphere.

In the following, several retrieved temperature profiles are compared with the corresponding profiles which were calculated by scientists at JPL (95-07-01-ecmwfformat data) and UCAR (GPS/MET database) by using the same occultation data and a similar retrieval. Contrary to UCAR and IMG (Institute for Meteorology and Geophysics, this study), the retrieval by JPL takes water vapor into account. This produces large differences in the retrieved temperature at heights below $10 \mathrm{~km}$.

In Fig. 6 the agreement between JPL and IMG is quite good at heights between 8 and $40 \mathrm{~km}$ : the difference is less than $2 \mathrm{~K}$. The profile of UCAR shows larger differences at heights beyond $25 \mathrm{~km}$. This may be due to the different choice of the initial heights for the Abelian integral inversion: JPL always starts at $50 \mathrm{~km}$ height, UCAR starts at heights around $60 \mathrm{~km}$ while IMG used the statistical optimization method which produces almost the same results for different initial heights. In Fig. 7 the UCAR and IMG profiles are in good agreement above $15 \mathrm{~km}$ height while the JPL profile has a shift to lower temperatures. Over the height range 5-15 km JPL and IMG are in better agreement. An example of an extreme disagreement between the retrievals of JPL, UCAR and IMG is depicted in Fig. 8. The noticeable deviation of the JPL profile relative to UCAR and IMG at heights beyond $20 \mathrm{~km}$ may be caused by data runaways and noise between heights 40-50 km which were perhaps not removed by the JPL retrieval. The IMG and UCAR profiles show differences of around $5 \mathrm{~K}$, so that the agreement is also poor.

The comparison shows that uncertainty exists in the retrieval of the GPS/MET data which is due to the occurrence (and treatment) of noise and errors in the phase measurements, in particular at heights beyond $40 \mathrm{~km}$. The disturbing noise and errors are mainly caused by ionospheric influences which become important at higher altitudes (Fig. 2). 


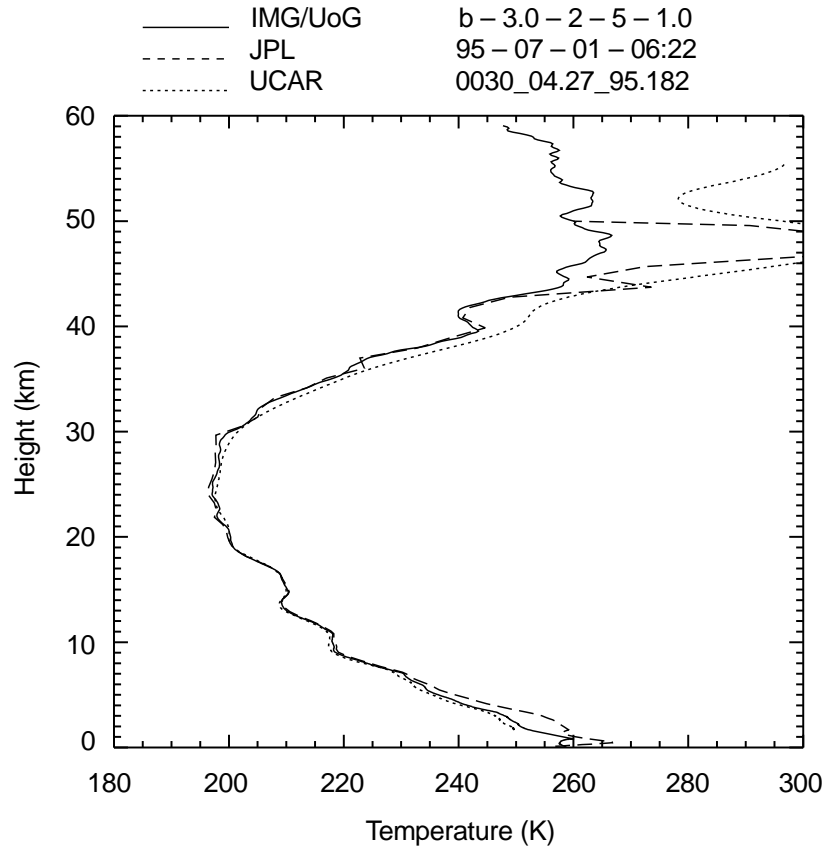

Fig. 6. Comparison of GPS/MET temperature profiles retrieved by IMG (solid line, retrieval from UCAR level2 data), by scientists of the Jet Propulsion Laboratory (JPL, dashed line, ecmwf-format data file) and by the Universities Corporation for Atmospheric Research (UCAR, dotted line, GPS/MET database). The occultation event (0030-04.27-95.182) was placed at $62^{\circ} \mathrm{S}, 144^{\circ} \mathrm{W}$ on 1 July $1995,6: 22 \mathrm{UT}$

\subsection{Wavelike structures}

In this section the potential of GPS/MET for observation of atmospheric waves is discussed. The interpretation of periodic structures in GPS/MET temperature profiles by atmospheric waves is dangerous at heights above $30 \mathrm{~km}$,

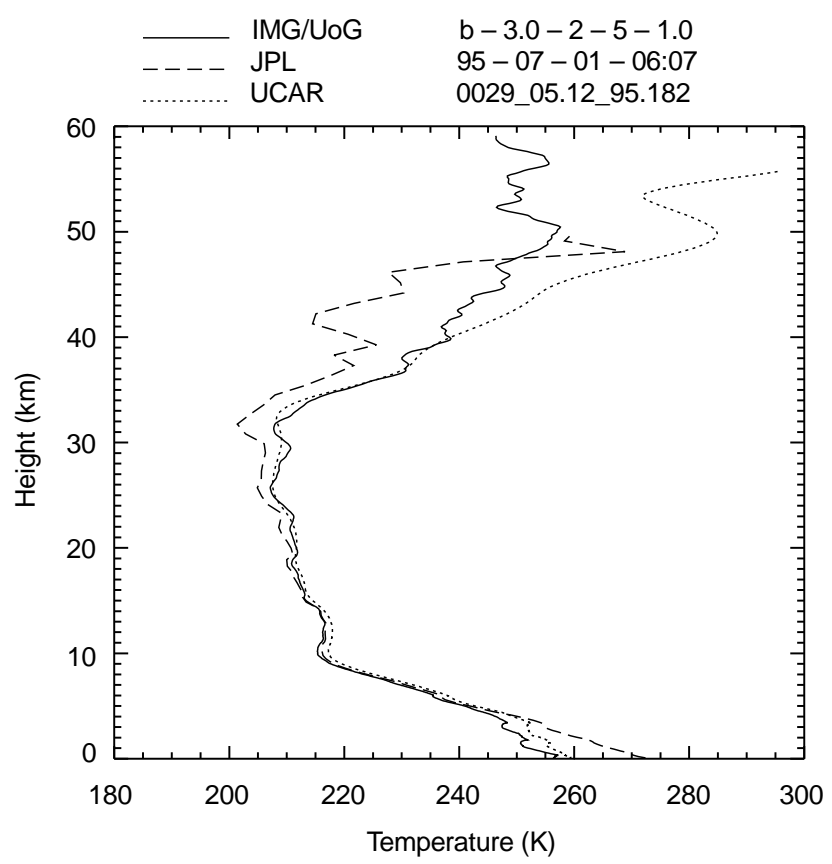

Fig. 7. Same as Fig. 6 but for the occultation event placed at $53^{\circ} \mathrm{S}$, $100^{\circ} \mathrm{E}$ on 1 July $1995,6: 07$ UT

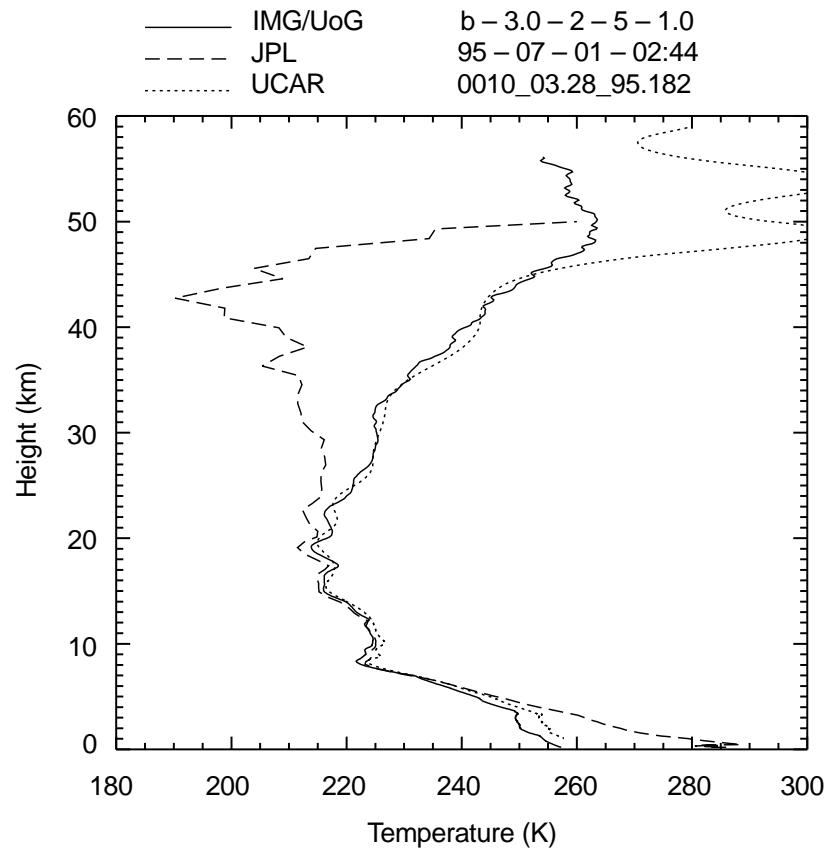

Fig. 8. Same as Fig. 6. This is an example for extreme disagreement between the three temperature retrievals. The occultation event took place at $37^{\circ} \mathrm{S}, 156^{\circ} \mathrm{E}$ on 1 July $1995,2: 44 \mathrm{UT}$

since the retrieval of simulation data showed that temperature oscillations were produced at this height region by the residual ionospheric error of the linear correction method. The oscillations occurred if the radio ray propagated through a non-spherical model ionosphere (without any fluctuations but with small discontinuities) and through a waveless neutral atmosphere. In the case of a spherical model ionosphere, which was strictly continuous, the oscillations of the retrieved temperature profile disappeared (Syndergaard private communication 1996). This retrieval error is due to the imperfect treatment of the splitting of the $f_{1}$ and $f_{2}$ ray paths by the ionospheric correction method. Below 30 $\mathrm{km}$ the relative contribution of the residual ionospheric error decreases exponentially and causes only a temperature error which is less than $1 \mathrm{~K}$, even for a daytime ionosphere at solar maximum (Kursinski et al., 1996b), so that in the troposphere and lower stratosphere the influence of the residual ionospheric error is negligible.

While the occultation data have a high vertical resolution between ca. $0.5 \mathrm{~km}$ (near the ground) and $1.5 \mathrm{~km}$ (above $20 \mathrm{~km}$ ), the horizontal resolution is between 160 and $280 \mathrm{~km}$, respectively (Hajj et al., 1995). Ray tracing simulations by Belloul and Hauchecorne (1996) for model atmospheres with periodic inhomogeneities (like those produced by gravity waves) showed that the occultation method is most sensitive to waves with horizontal wavelengths greater than $300 \mathrm{~km}$. Waves with smaller horizontal scales are smoothed, at least if the ray path is parallel to the horizontal wave vector. Therefore, the occultation method is able to measure fluctuations produced by equatorial waves or large-scale gravity waves. Radio occultation data for a system of several LEO satellites and, in supplement, 


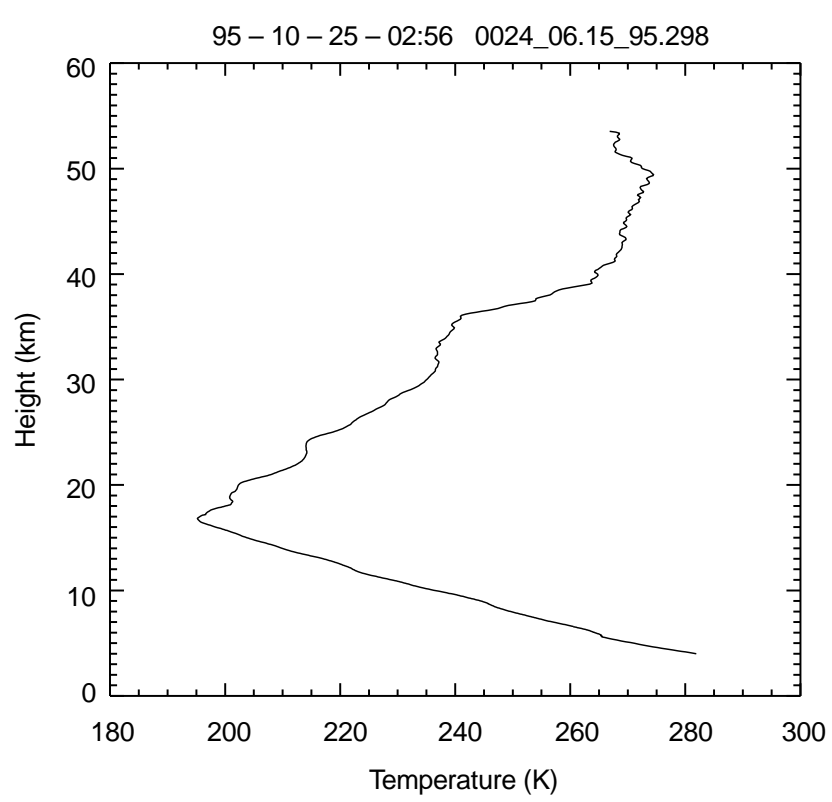

Fig. 9. Wavelike structures in a tropical temperature profile at tropospheric and stratospheric heights (25 Oct. 1995, 2:56 UT, ${ }^{\circ} \mathrm{S}$, $\left.91^{\circ} \mathrm{W}\right)$

data of high-resolution radiosondes, which can measure waves with smaller horizontal scales at heights below $30 \mathrm{~km}$ (Hamilton and Vincent, 1995), promise to give a detailed description of tropospheric and stratospheric dynamics.

In the equatorial lower stratosphere, many of the retrieved temperature profiles showed wavelike structures which were interpreted as Rossby-gravity waves (Kursinski et al., 1996a; Ware et al. 1996). Kursinski et al. (1996a) presented one example of a GPS/MET temperature fluctuation which was in excellent agreement with the measurement of a nearby radiosonde. Both temperature profiles showed a wave with a vertical wavelength of around $3 \mathrm{~km}$ in the lower stratosphere.

The temperature profile of Fig. 9 was measured on 25 October 1995 in the tropical zone $\left(2: 56 \mathrm{UT}, 12^{\circ} \mathrm{S}, 91^{\circ} \mathrm{W}\right)$ and shows a great variety of periodic structures. In the lower stratosphere $(h=18-25 \mathrm{~km})$ a wave with a vertical wavelength of around $4 \mathrm{~km}$ is visible which looks like the Rossby-gravity wave described by Kursinski et al. (1996a). In the troposphere (below $17 \mathrm{~km}$ ) a wavelike structure with a smaller amplitude is visible over several cycles. Between 30 and $40 \mathrm{~km}$ there is a strong deviation from the normal shape of the temperature profile in that region.

Another example of wavelike temperature structures in the troposhere and stratosphere is depicted in Fig. 10. The temperature profile was measured on 25 October $1995,3: 38 \mathrm{UT}$ at mid-latitudes $37^{\circ} \mathrm{N}, 66^{\circ} \mathrm{E}$.

\section{Summary}

The inversion of GPS/MET data was described with special consideration of the upper boundary condition. It was shown that the statistical optimization approach,

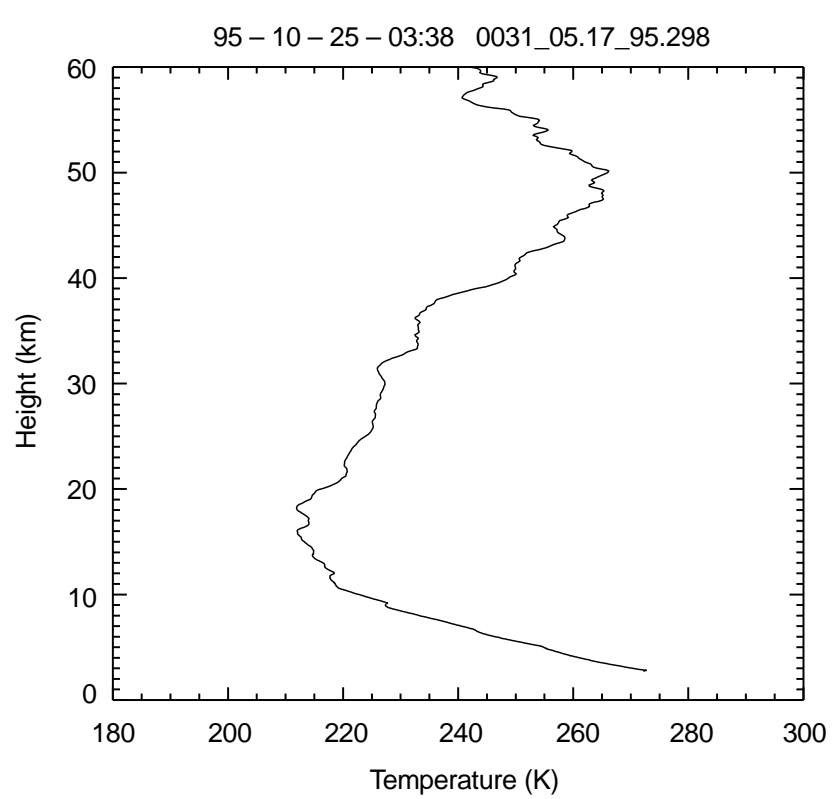

Fig. 10. Periodic structures in the temperature profile, especially in the height range $30-45 \mathrm{~km}$ but also below (25 Oct. 1995, 3:38 UT, $37^{\circ} \mathrm{N}$ lat, $\left.66^{\circ} \mathrm{E}\right)$

which was applied to the bending angle data between $40 \mathrm{~km}$ and the initialization height $(50-60 \mathrm{~km})$, stabilized the inversion by controlling the noise and residual ionospheric error of the bending angle in this height region. Beyond the initialization height a model bending angle was used which was calculated from the density profile of the MSISE-90 model at the place and time of the occultation.

The retrieval was compared with related retrievals of JPL and UCAR for several radio occultation events. The agreement was of different quality depending on the noise and residual ionospheric errors of the bending angles. A further refinement of the data analysis and development of ionospheric correction methods of higher order (Gorbunov et al., 1996) will improve the quality of the measured bending angle at higher altitudes and will shift the upper boundary of the Abelian integral inversion more upward. This will reduce the dependence of the retrieved temperature profile on the model bending angle profile. Furthermore it could be possible to fit the MSISE model bending angle profile towards to the unknown real bending angle profile of the individual occultation event by consideration of the observed density and temperature behavior at lower heights and by using knowledge of the coupling of troposphere, stratosphere and mesosphere (Leitinger private communication 1996).

Temperature profiles with various wavelike structures showed the potential of the radio occultation method for investigations of tropospheric and stratospheric dynamics.

Acknowledgements. I would like to thank S. Syndergaard from DMI for simulation data and valuable help. I am also grateful to A. Steiner from IMG for assistance in programming. G. Kirchengast from IMG is thanked for scientific-technical support. I wish to thank the GPS/MET principal investigator R. Ware, the project 
manager M. Exner and the GPS/MET team of the Universities Corporation for Atmospheric Research (UCAR), Boulder, Colorado for operating the GPS/MET experiment and supplying the data. I thank the Jet Propulsion Laboratory, California Institute of Technology for the retrieved temperature profiles on 1 July 1995. The work was funded by the ESA.

Topical Editor, L. Eymard thanks A. Hauchecorne and R. Vincent for their help in evaluating this paper.

\section{References}

Belloul, B., and A. Hauchecorne, The effect of gravity waves on occultation profiles, in DMI Scientific Report 95-4: Derivation of atmospheric properties using a radio occultation technique, 107-118, Copenhagen, Denmark, 1996.

Fjeldbo, G., and V. R. Eshelman, The bistatic radar-occultation method for the study of planetary ionospheres, J. Geophys. Res. 70, 3217-3225, 1965 .

Fjeldbo, G., A. J. Kliore, and V. R. Eshelman, The neutral atmosphere of Venus as studied with the Mariner $\mathrm{V}$ radio occultation experiments, Astron. J. 76, 123-140, 1971.

Gorbunov, M. E., Accuracy of the refractometric method in a horizontally nonuniform atmosphere, Izv., Atmos. and Ocean. Phys. 5, 381-384, 1988.

Gorbunov, M. E., and S. V. Sokolovskiy, Remote sensing of refractivity from space for global observations of atmospheric parameters, Rep. 119, Max-Planck-Institute for Meteorology, Hamburg, 1993.

Gorbunov, M. E., S. V. Sokolovskiy, and L. Bengtsson, Space refractive tomography of the atmosphere: modeling of direct and inverse problems, Rep., Max-Planck-Institute for Meteorology, Hamburg, 1996.

Gurvich, A. S., V. Kan, L. I. Popov, V. V. Ryumin, S. A. Savchenko, and S. V. Sokolovskiy, Reconstruction of the atmosphere's temperature profile from motion pictures of the Sun and Moon taken from the Salyut-6 orbiter, Izv., Atmos. Ocean. Phys. 1, $7-$ 13, 1982.

Hajj, G. A., E. R. Kursinski, W. I. Bertiger, S. S. Leroy, T. K. Meehan, L. J. Romans, and J. T. Schofield, Initial results of GPS-LEO occultation measurements of Earth's atmosphere obtained with the GPS-MET experiment, in Proceedings of the IAG Symposium G1: GPS trends in precise terrestrial, airborne, and spaceborne applications, IUGG XXI General Assembly, Boulder CO, July 2-14, 1995.
Hamilton, K., and R.A. Vincent, High-resolution radiosonde data offer new prospects for research, EOS, 76, (49), 497-506, 1995.

Hedin, A.E., Extension of the MSIS thermosphere model into the middle and lower atmosphere, J. Geophys. Res. 96, 1159-1172, 1991.

Hoeg, P., S. Syndergaard, A. Hauchecorne, B. Belloul, G. Kirchengast, R. Leitinger, and W. Rothleitner, Derivation of atmospheric properties using a radio occultation technique, DMI Scientific Report 95-4, Copenhagen, Denmark, 1996.

Kliore, A.J., D.L. Cain, G.S. Levy, V.R. Eshleman, G. Fjeldbo, and F.D. Drake, Occultation experiment: results of the first direct measurement of Mars' atmosphere and ionosphere, Science $\mathbf{1 4 9}$ 1243-1248, 1965.

Kursinski, E.R., G.A. Hajj, W.I. Bertiger, S.S. Leroy, T.K. Meehan, L.J. Romans, J.T. Schofield, D.J. McCleese, W.G. Melbourne, C.L. Thornton, T.P. Yunck, J.R. Eyre, and R.N. Nagatani, Initial results of radio occultation observations of Earth's atmosphere using the global positioning system, Science 271, 1107-1110, 1996a.

Kursinski, E.R., G.A. Hajj, K.R. Hardy, J.T. Schofield, and R. Linfield, Observing Earth's atmosphere with radio occultation measurements using GPS, J. Geophys. Res., in press, 1996b.

Melbourne, W.G., E.S. Davis, C.B. Duncan, G.A. Hajj, K.R. Hardy, E.R. Kursinski, T.K. Meehan, L.E. Young, and T.P. Yunck, The application of spaceborne GPS to atmospheric limb sounding and global change monitoring, JPL Publication 94-18, Pasadena CA, 1994.

Sokolovskiy, S.V., Solution of the inverse refraction problem by sensing of the atmosphere from the space, Sov. J. Remote Sensing 3, 333-338, 1990.

Sokolovskiy, S., and D. Hunt, Statistical optimization approach for GPS/MET data inversions, URSI GPS/MET Workshop, Tucson, Arizona, 1996.

Syndergaard, S., and P. Hoeg, Realistic radio occultation simulations using a $3 \mathrm{D}$ ray tracing model and subsequent inversion with the Abel transform, URSI GPS/MET Workshop, Tucson, Arizona, 1996.

Vorob'ev, V.V., and T.G. Krasil'nikova, Estimation of the accuracy of the atmospheric refractive index recovery from Doppler shift measurements at frequencies used in the NAVSTAR system, Phys. Atmos Ocean 29, 602-609, 1994.

Ware, R., M. Exner, D. Feng, M. Gorbunov, K. Hardy, B. Herman, Y. Kuo, T. Meehan, W. Melbourne, C. Rocken, W. Schreiner, S. Sokolovskiy, F. Solheim, X. Zou, R. Anthes, S. Businger, and K. Trenberth, GPS sounding of the atmosphere from low earth orbit: preliminary results, Bull. Am. Meteorol. Soc. 77, 19-40, 1996. 